\title{
Title: Early concepts of intimacy: young humans use saliva sharing to infer close relationships
}

\author{
Authors: Ashley J Thomas, ${ }^{1,2,4^{*}}$, Brandon Woo ${ }^{1,4}$, Daniel Nettle ${ }^{3}$, Elizabeth Spelke ${ }^{1,4}$, Rebecca
} $\mathrm{Saxe}^{2,4}$

\author{
Affiliations: \\ ${ }^{1}$ Department of Psychology, Harvard University; Cambridge, USA \\ ${ }^{2}$ Brain and Cognitive Sciences, MIT; Cambridge, USA \\ ${ }^{3}$ Population Health Sciences Institute; Newcastle University, Newcastle, UK \\ ${ }^{4}$ NSF Center for Brains, Minds and Machines; Cambridge, USA \\ *Corresponding author. Email: ajthomas@mit.edu
}

Abstract: Across human societies, people form 'thick' relationships, characterized by strong attachments, obligations and mutual responsiveness. People in thick relationships engage in distinctive interactions, like sharing food utensils or kissing, that involve sharing saliva. Here we show that children $(\mathrm{N}=113)$, toddlers $(\mathrm{N}=190)$, and infants $(\mathrm{N}=81)$ infer that dyads who share saliva (compared to other positive social interactions) have a distinct relationship. Children expect saliva sharing to happen in nuclear families. Toddlers and infants expect that people who shared saliva will respond to one another in distress. Parents $(\mathrm{N}=129)$ confirm that saliva sharing is a valid cue of relationship thickness in their children's social environments. The ability to use distinctive interactions to infer categories of relationships thus emerges early in life, without explicit teaching, allowing young humans to rapidly identify close relationships, both within and beyond families.

One-Sentence Summary: Children, toddlers and infants infer that individuals who share saliva are in distinctive close social relationships. 


\section{Main Text:}

In order to become a competent member of society, humans must learn how the people around them are related to each other (1-3). Across cultures, people distinguish a special category of relationships, which we will call 'thick' (4)(5-7), that feature strong levels of attachments, obligations, mutual responsiveness, and a feeling of oneness which is conceived in terms of shared bodily substance (5-7). Thick relationships are often, but not always, between close genetic relatives $(8-12)$. The fact that only some relationships are thick presents young humans with a problem: how do they identify which ones? For older children, distinct relationship categories can be explicitly verbally labelled (13). Anthropologists have claimed that younger children and even infants must be sensitive to how relationships are embodied in distinctive interactions $(14,15)$. For example, interactions that involve deliberate consensual transfer of saliva, such as kissing or sharing food utensils, distinctively occur in thick relationships $((16,17)$; see Supplemental Materials(18)). Here we apply experimental techniques from developmental science to test whether young children, toddlers, and infants do indeed infer that two individuals who share saliva are likely to be in a thick relationship.

In a first experiment, when presented with interactions between cartoon people, young children (Experiment 1, N=113, 5-7 years old, from an American urban environment) predicted that sharing utensils, or licking the same food item, would occur within nuclear families; whereas sharing toys and partitionable food would occur equally within friendships and families $(\chi 2=72.74, p<.001$, Figure 1; (19-21)). Thus young children recognize that saliva-sharing interactions distinctively occur within nuclear families. 


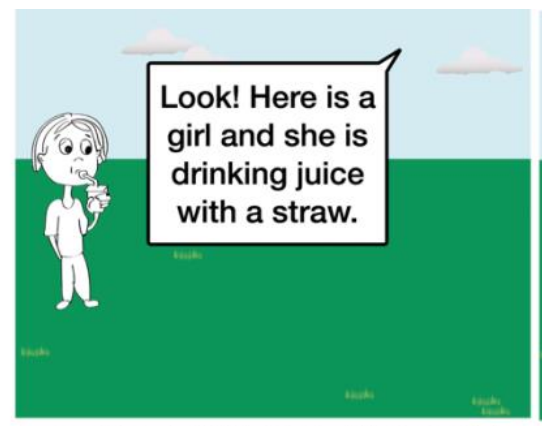

OBJECT INTRODUCTION

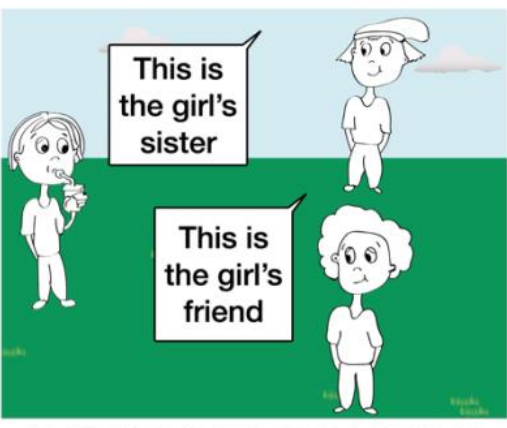

PEOPLE INTRODUCTION

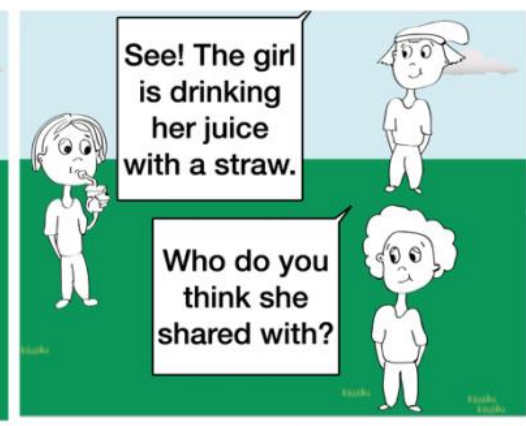

CHOICE

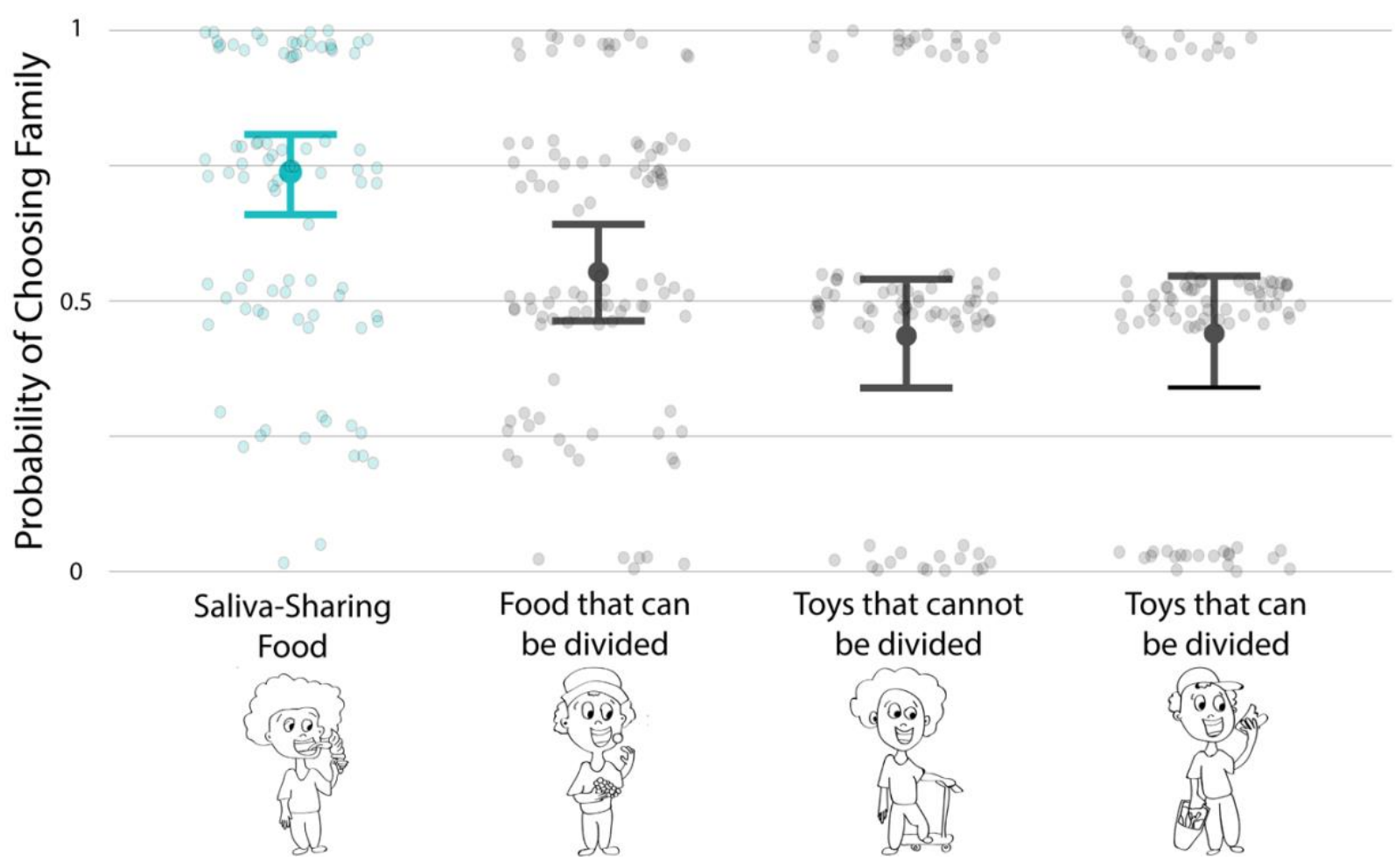

Fig. 1. Stimuli and Results for Experiment 1. (Top Panel): Sample images and text for one item of the storybook task. (Bottom Panel): Filled dots are average probability estimates of picking family as opposed to friend in each condition. The bars are 95\% credible intervals for each condition (controlling for multiple comparisons, and participant age). Unfilled dots are response rates from each individual child. Note that there were four items in each food condition and two items in each toy condition. 
In the next experiments, we tested whether toddlers and infants would predict that when two individuals have shared saliva, those individuals will be more emotionally responsive in future interactions (1). This experimental design was inspired by classic studies of vervet monkeys who heard a familiar juvenile in distress, and looked towards that juvenile's mother, as if expecting her to respond (22). We used this design to test whether young humans use a brief observation of saliva sharing to infer a thick relationship between novel individuals, whose genetic relatedness is unknown.

Toddlers (Experiment 2A, N=27, 16.5-18.5 months old) and infants (Experiment 2B, $\mathrm{N}=20,8.5-10$ months old) saw a central puppet alternately eat from the same orange slice with one actress (implying saliva sharing), and play ball with another actress (Figure 2). Then they saw the puppet seated between the two actresses, expressing distress. We measured which actress participants looked toward first, and longer, as though expecting the actress to react to the puppet's distress. Both toddlers and infants looked first, and longer, toward the actress who had shared food and saliva with the puppet (First look: 2 A Toddlers: 20/26; BF $10=10.796$; 2 B Infants: 16/20; $\mathrm{BF}_{10}=10.306$; Proportion look: $2 \mathrm{~A}$ Toddlers: $M=0.774 ; \mathrm{BF}_{10}=149.377 ; 2 \mathrm{~B}$ Infants: $M$ $\left.=0.675 ; \mathrm{BF}_{10}=6.084\right)$. These results were replicated in an independent sample of toddlers and infants (First look: $2 \mathrm{C}_{\text {test }}$ Toddlers: 19/23; $\mathrm{BF}_{10}=39.471 ; 2 \mathrm{D}_{\text {test }}$ Infants: $22 / 24 ; \mathrm{BF}_{10}=2431$; Proportion look: $2 \mathrm{C}_{\text {test }}$ Toddlers $M=0.788, \mathrm{BF}_{10}=3853 ; 2 \mathrm{D}_{\text {test }}$ Infants: $M=0.734, \mathrm{BF}_{10}=132$ ).

Further experiments revealed that toddlers and infants looked toward the food-and-salivasharing actress (i) only when the central puppet expressed distress and (ii) only when the puppet in distress was the actress' own thick relation. When the central puppet was removed leaving only the two actresses, toddlers and infants looked at both actresses equally (Figure 2 and (21)). When the central puppet was replaced by a new puppet, who then expressed distress, neither infants nor toddlers looked first or longer at the food sharer (First look: $2 C_{\text {control }}$ Toddlers: 12/28, 
$\mathrm{BF}_{01}=3.287 ; 2 \mathrm{D}_{\text {control }}$ Infants: $13 / 25 ; \mathrm{BF}_{01}=4.03$; Proportion look: Toddlers: $M=0.496$, $\mathrm{BF}_{01}=4.903$; Infants: $\left.M=0.470, \mathrm{BF}_{01}=4.46\right)$. These results suggest that toddlers' and infants' expectations concern the relationship, not the individuals' traits.

For experiment 2.E, we recruited a larger, more economically, geographically and racially diverse sample of toddlers $(\mathrm{N}=118$; age 14.5-19 months, see (21)). The familiarization events were the same. Then, the central puppet either expressed distress as before ( $\left.2 \mathrm{E}_{\text {test }}\right)$ or asked for the ball (2 $\left.\mathrm{E}_{\text {control }}\right)$. When the central puppet expressed distress, toddlers looked first, and longer, toward the food-sharer rather than the ball-passer (2Etest: First look: 35/48; $\mathrm{BF}_{10}=59.5$; Proportion look: $\left.M=0.65 ; \mathrm{BF}_{10}=1002\right)$. By contrast, when the puppet requested the ball, toddlers looked first and longer at the ball-passer (First look: $2 \mathrm{E}_{\mathrm{control}}: 13 / 52 ; \mathrm{BF}_{10}=267$; Proportion look: $\left.M=0.37 ; \mathrm{BF}_{10}=54\right)$. These conditions differed decisively (First look: $\mathrm{BF}_{10}$ $>1000$; proportion of time: $\mathrm{BF}_{10}>1000$ ). Thus, toddlers from a diverse range of households expect that two people who share food and saliva will respond to each other's distress, but not that they will be socially more responsive to one another in general. 
FAMILIARIZATION (8 TRIALS)

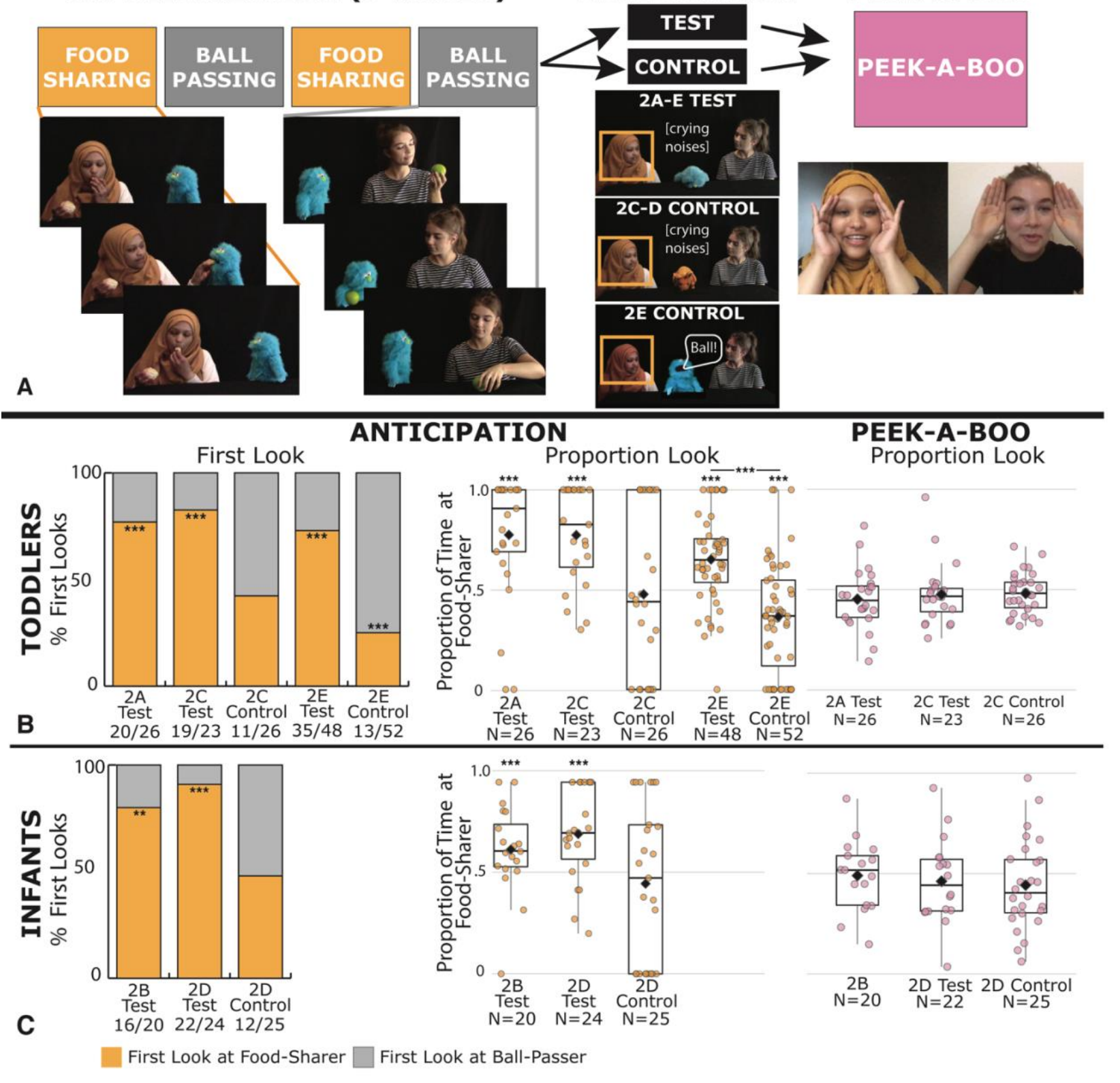

Fig 2. Stimuli and Results for Experiment 2. (A): Experimental design flowchart and stills from videos used in Experiment 2. The order of the familiarization trials (i.e., food-sharing or ball passing first) and the identity of the food-sharer were counterbalanced across participants. Participants were randomly assigned to the test or control condition. (B and C): Left bar plots show a percentage of participants who looked first toward the food-sharer (orange) or ball passer (grey). Middle panel plots are the proportion of time spent looking at the food-sharer during the pause. The black diamonds are means and bars are medians. The far right plot shows the 
proportion of time that participants spent looking at the food-sharer during the peek-a-boo trial. *** indicates a Bayes Factor of over 10. ** Indicates a Bayes Factor of over 8.

In Experiment 3, we isolated sharing saliva, without food, as the visible evidence of a thick relationship. Interacting with one puppet, a central actress put her finger in her own mouth, rotated it, put her finger in the puppet's mouth, rotated it, and finally returned her finger to her own mouth. When interacting with a second puppet, the actress performed the same rotating finger actions touching her own and the puppet's forehead. We then measured which puppet infants and toddlers looked toward, when the central actress expressed distress.

Toddlers (age 16.5-18.5 months) looked first, and longer, towards the puppet from the mouth-to-mouth interaction, when the actress expressed distress (Experiment 3A, N=27, First look: 20/26; $\mathrm{BF}_{10}=10.796$; Proportion look: $\left.M=0.746, \mathrm{BF}_{10}=477.6\right)$. These results were replicated in an independent sample of toddlers $\left(3 \mathrm{C}_{\text {test }} \mathrm{N}=31\right.$, First Look: $25 / 31, \mathrm{BF}_{10}=91.15$; Proportion look: $M=0.748, \mathrm{BF}_{10}=14856$ ). Infants' (age 8.5-10 months) first looks were distributed equally between both puppets (3B N=21, First look: $11 / 21$; $\mathrm{BF}_{01}=3.700$; replication $3 \mathrm{D}_{\text {test }} \mathrm{N}=26 ; 14 / 26$; $\left.\mathrm{BF}_{01}=3.886\right)$. Yet infants did look longer toward the puppet from the mouth-to-mouth interaction while the actress expressed distress $\left(3 \mathrm{~B} M=0.631 ; \mathrm{BF}_{10}=1.55\right.$; replication $3 \mathrm{D}_{\text {test }}: M=0.716$, $\mathrm{BF}=26.812)$.

Further experiments revealed that toddlers and infants looked toward the mouth-to-mouth puppet (i) only when the actor in distress was the person in the initial interactions, and (ii) only when the central actress expressed distress. When the actress was removed and replaced by a new actor who expressed distress, infants and toddlers seemed to expect that the other puppet, who had the forehead-to-forehead interaction with the first actress, was more likely to react 


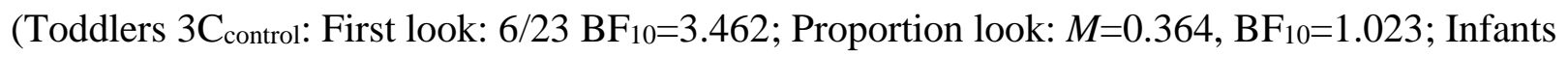

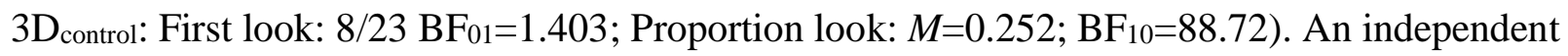
group of toddlers (Experiment 3E, (21)) looked toward the mouth-to-mouth puppet when the central actress expressed distress (3E $\mathrm{E}_{\text {test }}$ First look: 40/52; $\mathrm{BF}_{10}=823$; Proportion look: $M=0.690$, $\left.\mathrm{BF}_{10}=71669\right)$, but not when the actress uttered a nonsense word (3Econtrol First look:18/45; $\mathrm{BF}_{01}=2.43$; Proportion look: $\left.M=0.426 ; \mathrm{BF}_{01}=0.84\right)$. These two conditions differed decisively (First look: $\mathrm{BF}_{10}=418$; Proportion look: $\mathrm{BF}_{10}>1000$ ). Thus, toddlers from a wide range of households expect saliva sharing to selectively predict responses to distress. 

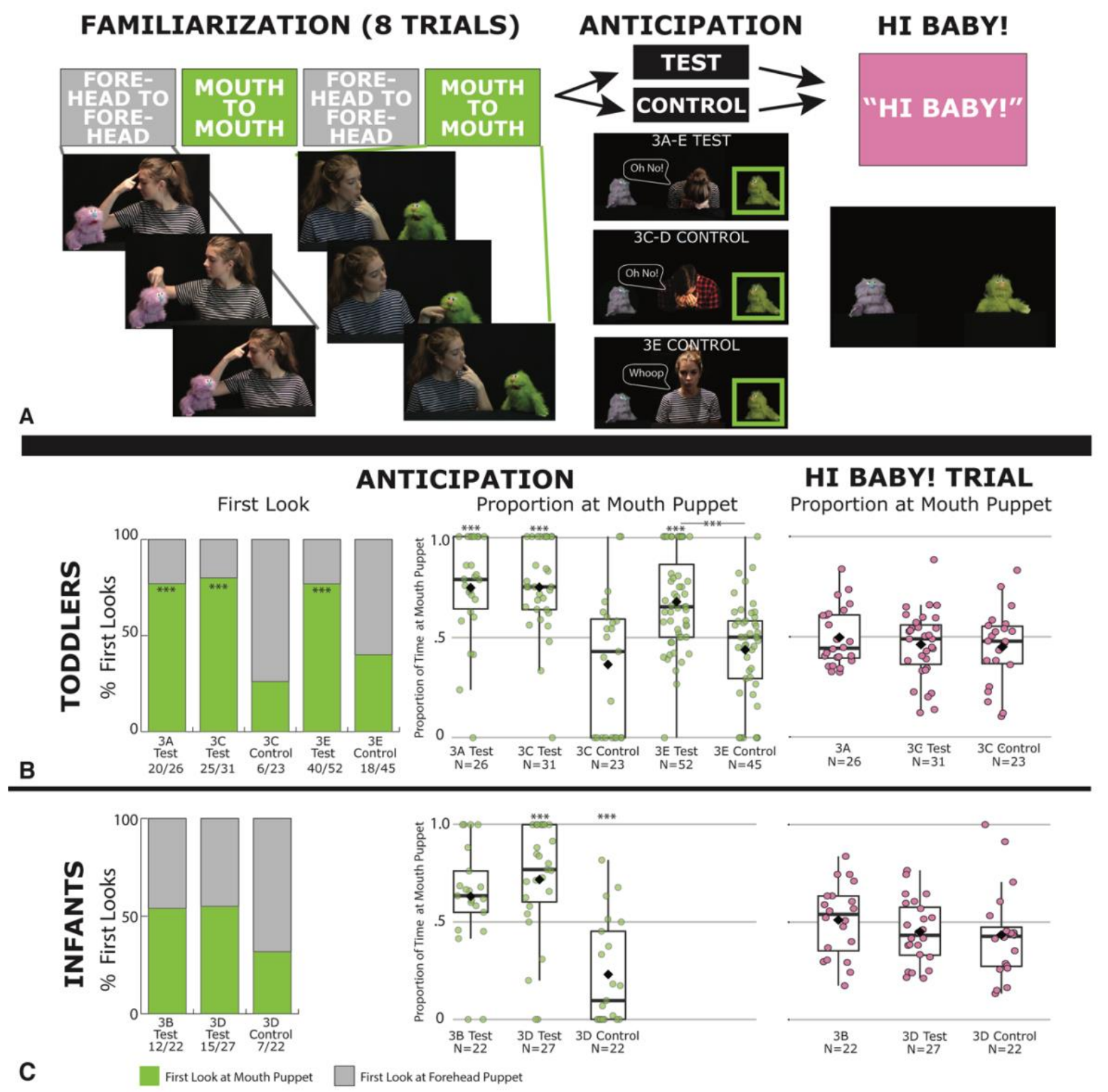

Fig 3. Stimuli and Results for Experiment 3. (A) Experimental design flowchart and stills from videos used in Experiment 3. (B and C): Left bar plots show percentage of participants who looked first toward the puppet who had engaged in the mouth-to-mouth interaction (green) or forehead-to-forehead interaction (grey). Middle box plots are the proportion of time spent looking at the mouth-to-mouth puppet during the pause. The black diamonds are means and bars are medians. The right box plots show the proportion of time that participants spent looking at the mouth-to-mouth puppet during the 'hi baby, hi' trial. *** indicates a Bayes Factor of over 10. 
The results of Experiments 2 and 3 suggest that when toddlers and infants observe two unfamiliar individuals share saliva, they infer that those people are in a thick relationship. A separate survey of parents ( $\mathrm{N}=129$, Experiment 4,(21)) of infants and toddlers (age 8-19 months) from the same population suggested that this inference would be valid. The parents expressed comfort with their child having positive social interactions (e.g. playing, reading, hugging) with people in many different relationships; but they expressed comfort with saliva-sharing interactions (i.e. sharing a utensil, drinking from the same cup, kissing on the face) only in relationships the participants assessed as thick (BF>1000; Figure 4). In summary, saliva-sharing interactions provide externally observable cues of thick relationships, and young humans can use these cues to make predictions about subsequent social interactions. 


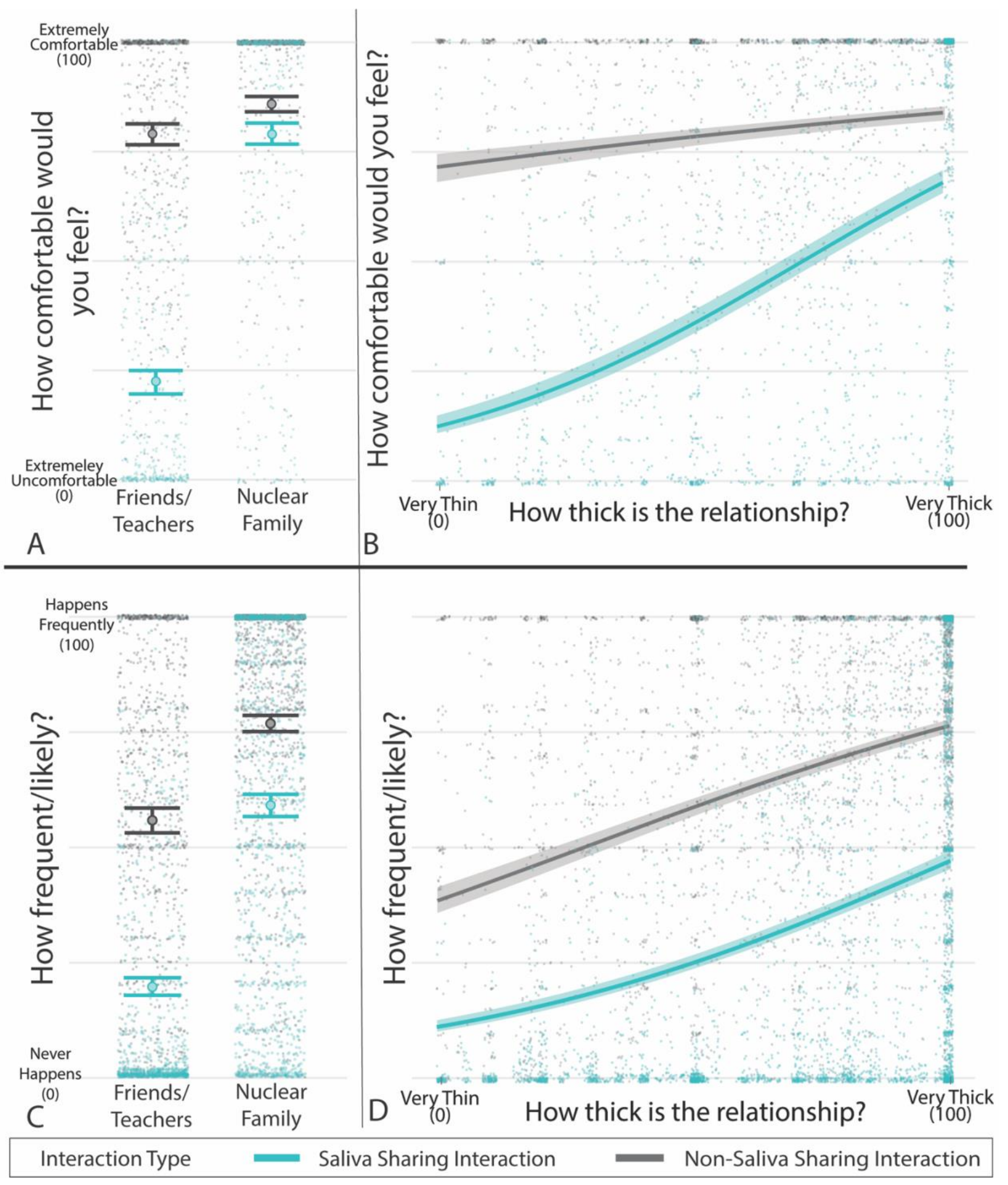

Fig 4. Results for Experiment 4. Parent-reported comfort $(\mathbf{A}, \mathbf{B})$ and frequency $(\mathbf{C}, \mathbf{D})$ of salivasharing interactions, versus other caregiving and prosocial interactions, in their child's social environment, as a function of relationship thickness. We operationalize relationship thickness as 
nuclear family (vs friends and teachers, A,C), and by directly asking parents to report the thickness for many of the child's relationships (e.g. including aunt, grandparent, etc; B,D).

Substantial prior research has shown that infants have an "intuitive psychology," supporting inferences about individuals' traits (e.g. cooperative, fair) (23-25), mental states (e.g. goals, perceptions) (26-28), and group membership (e.g., ethnicity, language) (29-33). By contrast, representations of social relationships and social structures, which are a central concern of sociology and anthropology, have been relatively understudied using experimental techniques in infants (34-36). The key distinction revealed here, between thick and thin relationships, may be one foundation of infants" "intuitive sociology" $(1,2)$.

School-aged children's judgments about saliva sharing are likely reinforced by explicit prohibitions (particularly during a pandemic), but similar intuitions appear to originate earlier and to generalize beyond the content of verbal rules motivated by hygiene $((37))$. We hypothesize that an early intuitive distinction between thick and thin relationships allows infants to rapidly learn the distinctive behaviors that occur in these relationships in their social environment $(14,15)$. These rapidly bootstrapped representations would be useful for parsing the small set of thick, intimate relationships from the larger set of thin, cooperative relationships in typical human social networks $(14,15)$.

The current experiments have limitations. We have not established whether thick and thin relationships are conceived as qualitatively distinct categories (e.g. $(5,7,38)$, or as ends of a continuum, with close friends or confidants having a mixture of features $(39,40)$. Saliva sharing is likely only one example of the set of interactions that children and infants can use to distinguish thick relationships from other cooperative relationships. Interactions distinctively occurring in thick relationships include confiding and emotional comforting, consensual 
exchange of blood, and touching genitals (15). Infants may also have expectations about the social categories of the people in thick relations (for example, older women may be more likely to be in thick relationships with infants than older men (41)). Conversely, some interactions involving transfer of saliva are aggressive and demeaning, like spitting on a person(18). We have not established whether toddlers and infants can distinguish between saliva sharing interactions that are consensual versus coercive.

Our evidence that young children, toddlers and infants make distinctive inferences about thick relationships has broad theoretical implications. Anthropologists have stressed that thick relationships, characterized by strong attachment obligation and mutual responsiveness, have highly variable mappings onto genetic relatedness $(10,42)$. Here we identify a proximal psychological mechanism that might allow infants to parse this variability $(1,3)$. A consistent inferential mechanism, operating in diverse social and ecological settings, could enable young humans to rapidly acculturate to their local and culturally-specific relationship and kinship structures. In sum, children, toddlers, and infants recognize saliva-sharing interactions as distinctive evidence of a thick relationship. The pattern of who does, and who does not, share saliva may help infants to distinguish among their many caregivers $(43,44)$, those who are kin

(e.g. parents, siblings, grandparents) versus non-kin (e.g. daycare teachers, nannies). Young humans may use observations of saliva sharing to inform their earliest understanding of the conceptual structure of family.

\section{References and Notes}

1. L. Kaufmann, F. Clément, Wired for Society: Cognizing Pathways to Society and Culture. Topoi (2014), doi:10.1007/s11245-014-9236-9.

2. L. Thomsen, S. Carey, in Navigating the Social World: What Infants, Children, and Other Species Can Teach Us (Oxford University Press, 2013), p. 17. 
3. L. A. Hirschfeld, On a Folk Theory of Society: Children, Evolution, and Mental Representations of Social Groups. Pers. Soc. Psychol. Rev. 5, 107-117 (2001).

4. A. Margalit, On Betrayal (Harvard University Press, 2017).

5. M. S. Clark, J. Mills, Interpersonal attraction in exchange and communal relationships. Journal of Personality and Social Psychology. 37 (1979), pp. 12-24.

6. T. S. Rai, A. P. Fiske, Moral psychology is relationship regulation: moral motives for unity, hierarchy, equality, and proportionality. Psychol. Rev. 118, 57-75 (2011).

7. M. Sahlins, Stone Age Economics (Taylor \& Francis, 2017).

8. D. M. Schneider, A Critique of the Study of Kinship (University of Michigan Press, 1984).

9. M. D. Sahlins, The Use and Abuse of Biology: An Anthropological Critique of Sociobiology (University of Michigan Press, 1976).

10. D. Jones, The generative psychology of kinship: Part 1. Cognitive universals and evolutionary psychology. Evol. Hum. Behav. 24, 303-319 (2003).

11. L. Cronk, D. Steklis, N. Steklis, O. R. van den Akker, A. Aktipis, Kin terms and fitness interdependence. Evol. Hum. Behav. 40, 281-291 (2019).

12. J. Carsten, the substance of kinship and the heat of the hearth: feeding, personhood, and relatedness among Malays in Pulau Langkawi. Am. Ethnol. 22, 223-241 (1995).

13. L. A. Hirschfeld, Rethinking the Acquisition of Kinship Terms. Int. J. Behav. Dev. 12, 541568 (1989).

14. D. Cohen, A. K.-Y. Leung, The hard embodiment of culture. Eur. J. Soc. Psychol. 39, 1278-1289 (2009).

15. A. P. Fiske, L. Thomsen, S. M. Thein, Differently embodying different relationships. Eur. J. Soc. Psychol. 39, 1294-1297 (2009).

16. A. Sorokowska, S. Saluja, P. Sorokowski, T. Frąckowiak, M. Karwowski, T. Aavik, G. Akello, C. Alm, N. Amjad, A. Anjum, K. Asao, C. S. Atama, D. Atamtürk Duyar, R. Ayebare, C. Batres, M. Bendixen, A. Bensafia, B. Bizumic, M. Boussena, D. M. Buss, M. Butovskaya, S. Can, K. Cantarero, A. Carrier, H. Cetinkaya, D. Chabin, D. Conroy-Beam, J. Contreras-Graduño, M. A. C. Varella, R. M. Cueto, M. Czub, D. Dronova, S. Dural, I. Duyar, B. Ertugrul, A. Espinosa, C. S. Esteves, F. Guemaz, M. Hal'amová, I. Herak, I. Hromatko, C.-M. Hui, J. L. Jaafar, F. Jiang, K. Kafetsios, T. Kavcic, L. E. O. Kennair, N. O. Kervyn, I. A. Khilji, N. C. Köbis, A. Kostic, A. Láng, G. R. Lennard, E. León, T. Lindholm, G. Lopez, Z. Manesi, R. Martinez, S. L. McKerchar, N. Meskó, G. Misra, C. Monaghan, E. C. Mora, A. Moya-Garofano, B. Musil, J. C. Natividade, G. Nizharadze, E. Oberzaucher, A. Oleszkiewicz, I. E. Onyishi, B. Özener, A. F. Pagani, V. Pakalniskiene, M. Parise, F. Pazhoohi, M. Pejičić, A. Pisanski, K. Pisanski, N. Plohl, C. Popa, P. Prokop, M. Rizwan, M. Sainz, S. Salkičević, R. Sargautyte, I. Sarmany-Schuller, S. Schmehl, A. 
Shahid, R. Shaikh, S. Sharad, R. S. Siddiqui, F. Simonetti, M. Tadinac, K. Ugalde González, O. Uhryn, C.-M. Vauclair, L. D. Vega Araya, D. A. Widarini, G. Yoo, Z. F. Zadeh, M. Zat'ková, M. Zupančič, I. Croy, Affective Interpersonal Touch in Close Relationships: A Cross-Cultural Perspective. Pers. Soc. Psychol. Bull., 146167220988373 (2021).

17. L. Miller, P. Rozin, A. P. Fiske, Food sharing and feeding another person suggest intimacy; two studies of American college students. Eur. J. Soc. Psychol. 28, 423-436 (1998).

18. An analysis of saliva sharing across cultures as described in ethnographies is available in the supplementary materials at the Science website.

19. K. R. Olson, E. S. Spelke, Foundations of Cooperation in Young Children. Cognition. 108, 1-9 (2008).

20. A. C. Spokes, E. S. Spelke, Children's Expectations and Understanding of Kinship as a Social Category. Front. Psychol. 7 (2016).

21. Materials and methods are available as supplementary materials at the Science website.

22. R. M. Seyfarth, D. L. Cheney, The structure of social knowledge in monkeys. Animal social complexity: Intelligence, culture, and individualized societies, 207-229 (2003).

23. J. K. Hamlin, Moral Judgment and Action in Preverbal Infants and Toddlers: Evidence for an Innate Moral Core. Curr. Dir. Psychol. Sci. 22, 186-193 (2013).

24. L. J. Powell, E. S. Spelke, Third-Party Preferences for Imitators in Preverbal Infants. Open Mind. 2, 61-71 (2018).

25. A. Geraci, L. Surian, The developmental roots of fairness: infants' reactions to equal and unequal distributions of resources. Dev. Sci. 14, 1012-1020 (2011).

26. A. Woodward, Infants selectively encode the goal object of an actor's reach. Cognition. 69, 1-34 (1998).

27. S. Carey, The Origin of Concepts (Oxford University Press, USA; 1 edition, 2009).

28. S. Liu, T. D. Ullman, J. B. Tenenbaum, E. S. Spelke, Ten-month-old infants infer the value of goals from the costs of actions. Science. 358, 1038-1041 (2017).

29. K. D. Kinzler, E. Dupoux, E. S. Spelke, The native language of social cognition. Proc. Natl. Acad. Sci. U. S. A. 104, 12577-12580 (2007).

30. Z. Liberman, A. L. Woodward, K. D. Kinzler, Preverbal Infants Infer Third-Party Social Relationships Based on Language. Cogn. Sci. 41, 622-634 (2017).

31. Y. Bar-Haim, T. Ziv, D. Lamy, R. M. Hodes, Nature and nurture in own-race face processing. Psychol. Sci. 17, 159-163 (2006).

32. L. J. Powell, E. S. Spelke, Preverbal infants expect members of social groups to act alike. 
Proceedings of the National Academy of Sciences. 110, E3965-72 (2013).

33. F. Ting, Z. He, R. Baillargeon, Toddlers and infants expect individuals to refrain from helping an ingroup victim's aggressor. Proc. Natl. Acad. Sci. U. S. A. 116, 6025-6034 (2019).

34. L. Thomsen, W. E. Frankenhuis, M. Ingold-Smith, S. Carey, Big and mighty: Preverbal infants mentally represent social dominance. Science. 331, 26-29 (2011).

35. A. J. Thomas, B. W. Sarnecka, Infants Choose Those Who Defer in Conflicts. Curr. Biol. (2019), doi:10.1016/j.cub.2019.05.054.

36. Z. Liberman, A. L. Woodward, K. R. Sullivan, K. D. Kinzler, Early emerging system for reasoning about the social nature of food. Proceedings of the National Academy of Sciences. 113, 9480-9485 (2016).

37. Additional data collected pre-pandemic are available in the supplementary materials at the Science website.

38. A. P. Fiske, The four elementary forms of sociality: framework for a unified theory of social relations. Psychol. Rev. 99, 689-723 (1992).

39. J. B. Silk, Cooperation without counting. Genetic and cultural evolution of cooperation, 37-54 (2003).

40. M. L. Small, Someone To Talk To (Oxford University Press, 2017).

41. H. Alvarez, Grandmother hypothesis and primate life histories. Am. J. Phys. Anthropol. 113, 435-450 (2000).

42. G. P. Murdock, Kin Term Patterns and Their Distribution. Ethnology. 9, 165-208 (1970).

43. S. B. Hrdy, J. M. Burkart, The emergence of emotionally modern humans: implications for language and learning. Philos. Trans. R. Soc. Lond. B Biol. Sci. 375, 20190499 (2020).

44. S. B. Hrdy, Mothers and Others. Natural History. 110 (2001), p. 50.

Acknowledgments: The authors thank Claire Lu, Shruti Ravikumar, Margot Austin, Vanessa Kudrnova, Sara Alansaari, William Pepe, Anne Harris, and Misha Oraa Ali for assistance with data collection and video coding. Thank you to Emily Chen for assistance with trimming videos, recruitment, and editing drafts of this manuscript. Thank you to Laura Schulz, Tomer Ullman, Shari Liu, Halie Olson, Lindsey Powell, and Michelle Hung for comments on earlier drafts of the 
manuscript. Statistical support was provided by data science specialist Steven Worthington at the Institute for Quantitative Social Science, Harvard University.

\section{Funding:}

NIH National Research Service; Award 1F32HD096829 (AJT)

Patrick J. McGovern Foundation (RS)

Guggenheim Foundation (RS)

Social Sciences and Humanities Research Council Doctoral Fellowship; Award 7522020-0474 (BW)

NSF Center for Brains Minds and Machines; Award Number CCF-1231216 (LS, AJT, BW, RS)

Siegel Foundation; Award S4881 (LS)

\section{Author contributions:}

Conceptualization: AJT RS ES DN

Methodology: AJT RS ES BW DN

Formal Analysis: AJT

Resources: ES RS

Investigation: AJT

Visualization: AJT

Funding acquisition: AJT RS ES

Project administration: AJT 
Supervision: AJT RS ES

Writing - original draft: AJT RS

Writing - review \& editing: AJT RS DN BW ES

Competing interests: Authors declare that they have no competing interests.

Data and materials availability: All data are available on OSF (https://osf.io/c69d5). A subset of participant videos are available on Databrary

https://nyu.databrary.org/volume/1253.

\section{Supplementary Materials}

Materials and Methods

Supplementary Text

Figs. S1

References (44-56) 
